Bull. Mater. Sci., Vol. 17. No. 6, November 1994, pp. 1071-1077. (C) Printed in India.

\title{
Gas permeabilities of bisphenol bridge-disubstituted polyarylates
}

\author{
U K KHARUL and S S KULKARNI* \\ Polymer Science and Engineering Group, Chemical Engineering Division, National Chemical \\ Laboratory, Pune 411008 , India
}

\begin{abstract}
This study reports the physical properties and gas permeation characteristics of polyarylates prepared by disubstitution of the bisphenolA bridge carbon. The results are compared with data from previously reported unsubstituted and mono-substituted polyarylates. Disubstitution is an effective method of changing the packing density, as measured by the d-spacing, and also increases the chain stiffness. The intersegmental spacing can be either decreased (by phenyl disubstitution) or significantly increased (by $-\mathrm{CF}_{3}$ disubstitution). Increased $d$-spacing in the case of $-\mathrm{CF}_{3}$ disubstitution increases permeabilities and reduces selectivities compared to the unmodified bisphenolA based polymer. The increased permeability and selectivity of the phenyl disubstituted polyarylates, in spite of the reduced d-spacing, supports an earlier finding that decreased bisphenol phenyl ring mobility tends to increase permeability.
\end{abstract}

Keywords. Permeability; polyarylates; gas separation.

\section{Introduction}

The economic viability of a membrane gas-separation process depends greatly on the permeation characteristics of the membrane material. The ideal membrane material would have high permeability $(P)$ coupled with high permselectivity $(\alpha)$ for the gas pair under consideration. Unfortunately, the trend observed with most materials shows a trade off, i.e. membrane materials which have high permeability have low selectivity and vice versa. A better understanding of the polymer structure-permeability relationship is required for synthesizing polymers with the desired permeation characteristics.

In order to take advantage of the naturally higher selectivities in glassy polymers, recent work has focussed on polymers such as polyimides (Kim et al 1988; Stern et al 1988; Coleman and Koros 1990), polycarbonates (Anand et al 1989; Schmidhauser and Longley 1990), polysulfones (McHattie et al 1991; Houde et al 1994a) and polyarylates (Sheu and Chern 1989; Charati et al 1991a). Polyarylates based on bisphenolA have been identified as having potentially higher permeabilities than other glassy polymers (Barbari et al 1989).

The structure of polyarylates can be modified by varying either the diol or acid monomers. For rational design, it is necessary to identify the effect of various structural modifications on the permeation characteristics.

In this context, recently, we prepared a series of polyarylates in which one methyl group on the bisphenol bridge carbon was systematically substituted by other groups, e.g. ethyl, isobutyl, phenyl and ethyl propionate (Houde et al 1994b).

\footnotetext{
*For correspondence.
} 
Testing of the polymers resulting from these monosubstituted bisphenols and a $1: 1$ mixture of iso and terephthalic acids showed two unusual results: (i) the packing density of these polymers as measured by their $d$-spacing (obtained from wide angle X-ray diffraction) or free volume (obtained from density measurements) did not vary significantly even though the methyl group was substituted by much bulkier groups and (ii) there was significant (3-fold) variation in the permeabilities of the various monosubstituted polymers. Surprisingly, increased permeability correlated well with decreased rotational mobility of the bisphenol phenyl rings (measured by the $\gamma$-transition in dynamic mechanical analysis). This result is contrary to theories (e.g. Pace and Daytner 1979) of gas permeation as well as previous interpretations (Light and Seymour 1982) of the permeability data in partially aliphatic polyarylates.

The present study is an attempt to build our understanding of the factors affecting permeation in substituted polyarylates. Molecular modelling (CharmM Quanta 2.1) indicates that these bridge carbon substituents are oriented towards the outside of the polymer chain helix. This is illustrated in figure 1 which shows minimized single chain conformation of a polyarylate prepared from isobutyl substituted bisphenolA (bisMIBK) and terephthalic acid in comparison with the unsubstituted polymer. The chain conformation in the vicinity of the bisphenol moiety is determined by the phenyl ring orientations. These orientations, in an energy minimized conformation, are determined by the repulsive interactions between the ortho hydrogens on the phenyl rings and the influence of the substituents on the bridge carbon (Charati et al 1991b). Based on the molecular modelling work, we hypothesized that if the bridge carbon was disubstituted, it would not be possible for the chain configuration to remain unaffected. If this was true, disubstitution might be an effective way of changing the polymer packing density, and thereby the permeation characteristics. Secondly, the disubstitution of the bridge carbon by rigid groups should lead to reduced segmental mobility and would be an additional data regarding the effect of this variable on permeability.

In this study, we describe the permeation characteristics of polyarylates based on two disubstituted bisphenols and iso- and terephthalic acids. The bisphenols used were: (i) phenyl disubstitution (bisBzp) and (ii) $-\mathrm{CF}_{3}$ disubstituted (bisF 6 ). The results are compared with the corresponding polymers based on bisphenolA and the mono-phenyl substituted bisphenol (bisAceto). The chemical structures and nomenclature of the polymers discussed here are shown in figure 2.

\section{Experimental}

\subsection{Material preparation}

BisBzP was prepared by following the general procedure outlined by Morgan (1970). In the first step, dichlorodiphenylmethane (DCDPM) was prepared by Friedel-Craft alkylation of $\mathrm{CCl}_{4}$ with benzene. To a stirred suspension of $\mathrm{AlCl}_{3}(10 \mathrm{~g}, 0.075 \mathrm{~mol})$ in $25 \mathrm{ml}$ of $\mathrm{CCl}_{4}, 11.7 \mathrm{~g}$ of benzene $(0.15 \mathrm{~mol})$ were added dropwise for $1 \mathrm{~h}$, maintaining the temperature at $5^{\circ} \mathrm{C}$. After the addition, the temperature of the reaction mixture was raised to ambient and left overnight. $25 \mathrm{ml}$ of $\mathrm{CH}_{2} \mathrm{Cl}_{2}$ were added to dilute the reaction mixture, which was then poured on crushed ice 


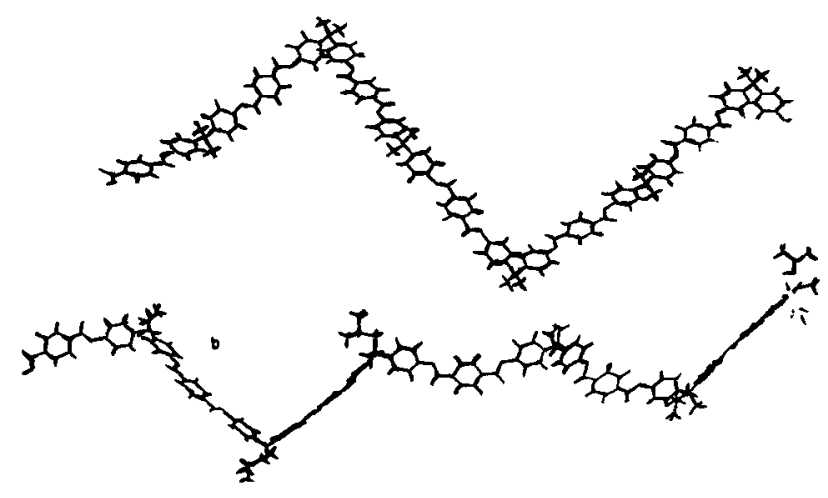

Figure 1. Representation of energy minimized single chain conformation for polyarylates based on terephthalic acid and (a) bisphenolA and (b) bisphenolA with isobutyl substitution, showing orientation of the bridge carbon substitution.<smiles>[R2]C([R2])(c1ccc(OC)cc1)c1ccc(OCc2ccc(C(C)=O)cc2)cc1</smiles>

\begin{tabular}{|c|c|c|c|}
\hline POLYMER & $R_{t}$ & $\mathbf{R}_{2}$ & $A C I D$ \\
\hline$B$ is $A-I$ & $-H$ & $-H$ & Iso \\
\hline Bis A - T & $-H$ & $-H$ & Tere \\
\hline Bis A-I + T & $-H$ & $-H$ & $(80+$ Tere $(1: 1)$ \\
\hline Bis Aceto-I+T & $-H$ & -0 & $1: 0+$ Tere $(1: 1)$ \\
\hline Bis $\theta 2 p-I+T$ & - & -(0) & $180+$ Tere $(1: 1)$ \\
\hline$B$ is $B 2 p-I$ & -0 & 우 & Iso \\
\hline Bis $F_{6}-I+T$ & $-C F_{3}$ & $-\mathrm{CF}_{3}$ & tso + Tere (t:1) \\
\hline
\end{tabular}

Figure 2. Chemical structure of polyarylates.

containing a few drops of concentrated $\mathrm{HCl}$. On stirring to break the $\mathrm{AlCl}_{3}$ complex, the organic layer separated and the aqueous layer was extracted twice with $\mathrm{CH}_{2} \mathrm{Cl}_{2}$. The combined organic layer was dried over $\mathrm{Na}_{2} \mathrm{SO}_{4}$ for about $3 \mathrm{~h}$ and the solvent was removed by distillation. Traces of the solvent were removed at low pressure. Thin layer chromatography of the resulting brown, viscous liquid showed only a trace amount of benzophenone impurity in the DCDPM.

In the second step, two molar equivalents of phenol were added to DCDPM. The reaction mixture was stirred overnight at $60^{\circ} \mathrm{C}$ and then heated at $170^{\circ} \mathrm{C}$ for 
around $5 \mathrm{~h}$ till it solidified. The bisphenol was dissolved in $\mathrm{NaOH}$ solution and reprecipitated by dilute $\mathrm{HCl}$ at $5^{\circ} \mathrm{C}$. This was further purified by recrystallization using tetrahydrofuran: cyclohexane. The yield of the pale yellow, shiny needles was found to be $60 \%$. The characterization was done by IR and $H^{\prime}$-NMR. (i) IR (nujol): $3600-3140 \mathrm{~cm}^{-1}$, broad, $-\mathrm{OH}$ stretch, $700 \mathrm{~cm}^{-1}, \mathrm{O}-\mathrm{H}$ out of plane deformation, $1620,1600,1520,1470,1450 \mathrm{~cm}^{-1}$, aromatic $\mathrm{C}=\mathrm{C}$ stretch, $1220-1000 \mathrm{~cm}^{-1}, m, \mathrm{C}-\mathrm{H}$ (aromatic) in plane bending and (ii) NMR; $\delta 3-3 \cdot 1, s$, hydroxyl protons, $\mathrm{D}_{2} \mathrm{O}$ exchangeable, $\delta 6 \cdot 75-6 \cdot 85, m, 4 \mathrm{H}$, aromatic, $\delta 6 \cdot 95-7 \cdot 1, m, 4 \mathrm{H}$, aromatic, $\delta 7 \cdot 15-7 \cdot 4$, $m, 10 \mathrm{H}$, aromatic.

BisphenolF $_{6} \quad$ [4,4'-(hexafluoroisopropyledene)diphenol], isophthalic acid and terephthalic acids were obtained from Aldrich Chemicals (USA) and used without further purification. Isophthaloyl dichloride (IPC) and terephthaloyl dichloride (TPC) were prepared by refluxing their respective acids in thionyl chloride using dimethyl formamide as a catalyst (0.013 molar equivalent for IPC and 0.08 for TPC). Both of them have almost quantitative yield and were characterized by melting point and IR.

\subsection{Polymer preparation and characterization}

Polymers were prepared by interfacial condensation using either IPC, TPC or their equimolar mixture. Polymers were purified by dissolving in chloroform and reprecipitating in acetone. The purified materials used for forming the polymer films were characterized by the following:

(i) Glass transition temperatures were determined on a Perkin-Elmer differential scanning calorimeter (model DSC-2C or DSC-7). The heating rate used was $20^{\circ} \mathrm{C} / \mathrm{min}$. (ii) The mean intersegmental spacing (d-spacing) was obtained as the length corresponding to the maximum of the amorphous diffraction pattern. Wide angle X-ray diffraction (WAXD) spectra were obtained on a Phillips PW 1730 X-ray generator unit in reflection geometry with $\mathrm{Cu}-\mathrm{K} \alpha$ radiation. The polymer samples were all amorphous with the exception of bisA-I.

(iii) Density measurements were done by the flotation method at $40^{\circ} \mathrm{C}$ using aqueous $\mathrm{K}_{2} \mathrm{CO}_{3}$ solution.

\subsection{Film preparation and permeation study}

Films of even thickness $(\sim 50 \mu)$ were prepared by solution casting from $5 \%$ chloroform solutions of the respective polymers. These films were vacuum dried at $65^{\circ} \mathrm{C}$ for at least eight days and then used for permeation studies. Permeabilities of the pure gases $\mathrm{He}, \mathrm{Ar}, \mathrm{N}_{2}, \mathrm{O}_{2}, \mathrm{CH}_{4}$ and $\mathrm{CO}_{2}$ were measured using the variable volume method. The measurements were done at, a high side gas pressure of $1 \times 10^{6} \mathrm{~N} / \mathrm{m}^{2}$ and $35^{\circ} \mathrm{C}$.

\section{Results and discussion}

The effects of disubstitution at the bisphenol bridge carbon are shown in table 1 which summarizes the data for polyarylates prepared from 4 bisphenols viz, bisA, 
bisAceto, bisBzp and bisF $_{6}$ with a $1: 1$ mixture of iso and terephthalic acids. It was previously mentioned that packing density does not change with monosubstitution at the bridge carbon as illustrated by the data for bisAceto-I+T in comparison with bisA-I+T (Houde et al 1994b). In contrast, both the disubstituted polymers viz. bisBzp-I+T and bisF $F_{6}-\mathrm{I}+\mathrm{T}$ show marked changes in packing density as measured by the d-spacing. In the case of the bisBzp based polymer, the decrease in d-spacing to $4.7 \AA$ may be attributed to increased interchain attraction. Such behaviour has been seen before with polar substitutions (e.g. Muruganandam et al 1987). In the case of the bisF $_{6}$ polymer, the effect is reversed and increased interchain repulsion appears to be responsible for a significant increase in d-spacing to $6.51 \AA$.

It is generally accepted that the packing density is one of the most important variables affecting the permeability (Stern et al 1989; Charati et al 1991a). It has been shown that the d-spacing obtained from WAXD data is generally a sensitive and accurate determinant of the permeability of the polymer (Charati et al 1991a). In this context, the 3-4 fold increase in permeability for bis $\mathrm{F}_{6}-\mathrm{I}+\mathrm{T}$ compared to bisA-I+T is in accordance with the increased d-spacing in the case of bis $F_{6}-I+T$. The decreased selectivity is also a consequence of the increased d-spacing which reduces the ability of the polymer matrix to discriminate between various gas molecules on the basis of size.

In the same context, it is interesting to compare the data for bisBzp-I+T with bisA-I+T and bisAceto-I+T. Both bisBzp-I+T and bisAceto-I+T have very similar permeabilities and selectivities. In both cases the permeabilities and selectivities are $30-50 \%$ higher than for bisA-I+T. This increase can be attributed to the decreased rotational mobility of the bisphenol phenyl rings. This correlation was shown earlier in the case of mono-substituted polymers where the d-spacing did not change markedly within the series (Houde et al 1994b). The correlation is even

Table 1. Permeation data and physical parameters of various polyarylates .

\begin{tabular}{llllll}
\hline Property & $\begin{array}{l}\text { BisA- } \\
\text { I+T }\end{array}$ & $\begin{array}{c}\text { BisAceto- } \\
\text { I+T }\end{array}$ & $\begin{array}{l}\text { BisBzp- } \\
\text { I+T }\end{array}$ & $\begin{array}{l}\text { BisBzp- } \\
\text { I }\end{array}$ & $\begin{array}{l}\text { BisF } \\
\text { I+T }\end{array}$ \\
\hline d-Spacing & 5.15 & 5.09 & 4.72 & 4.76 & 6.51 \\
Density & 1.212 & 1.211 & 1.217 & 1.221 & 1.412 \\
Tg & 198 & 229 & 251 & 229 & $233^{d}$ \\
$P(\mathrm{He})^{b}$ & 16 & 16 & 18 & 16 & 51 \\
$\mathrm{P}(\mathrm{Ar})$ & 0.56 & 0.74 & 0.79 & 0.48 & 2.6 \\
$\mathrm{P}\left(\mathrm{N}_{2}\right)$ & 0.33 & 0.32 & 0.33 & 0.23 & 1.5 \\
$\mathrm{P}\left(\mathrm{O}_{2}\right)$ & 1.4 & 2.1 & 1.7 & 1.3 & 5.6 \\
$\mathrm{P}\left(\mathrm{CH}_{4}\right)$ & 0.4 & 0.48 & 0.31 & 0.18 & 1.35 \\
$\mathrm{P}\left(\mathrm{CO}_{2}\right)$ & 7.3 & 11 & 11 & 8.4 & 35 \\
$\alpha\left(\mathrm{He}_{2}\right)^{c}$ & 48.5 & 49.7 & 53.3 & 69.6 & 34.3 \\
$\alpha\left(\mathrm{O}_{2} / \mathrm{N}_{2}\right)$ & 4.2 & 6.6 & 5.3 & 5.5 & 3.8 \\
$\alpha\left(\mathrm{CO}_{2} / \mathrm{CH}_{4}\right)$ & 18.3 & 22.5 & 35.2 & 46.7 & 25.8 \\
$\alpha\left(\mathrm{N}_{2} / \mathrm{CH}_{4}\right)$ & 0.83 & 0.67 & 1.06 & 1.28 & 1.10 \\
\hline
\end{tabular}

${ }^{a}$ Polymer nomenclature as given in figure 2 ; ${ }^{b}$ Permeability in units of $10^{-10}\left[\mathrm{~cm}^{3}(\mathrm{STP}) \mathrm{cm} / \mathrm{cm}^{2}\right.$.sec.cm $\left.\mathrm{Hg}\right]$ measured at $35^{\circ} \mathrm{C}$ and $1 \times 10^{6} \mathrm{~N} / \mathrm{m}^{2}$; Selectivity is the ratio of pure gas permeabilities; 'Maruyama et al (1986). 

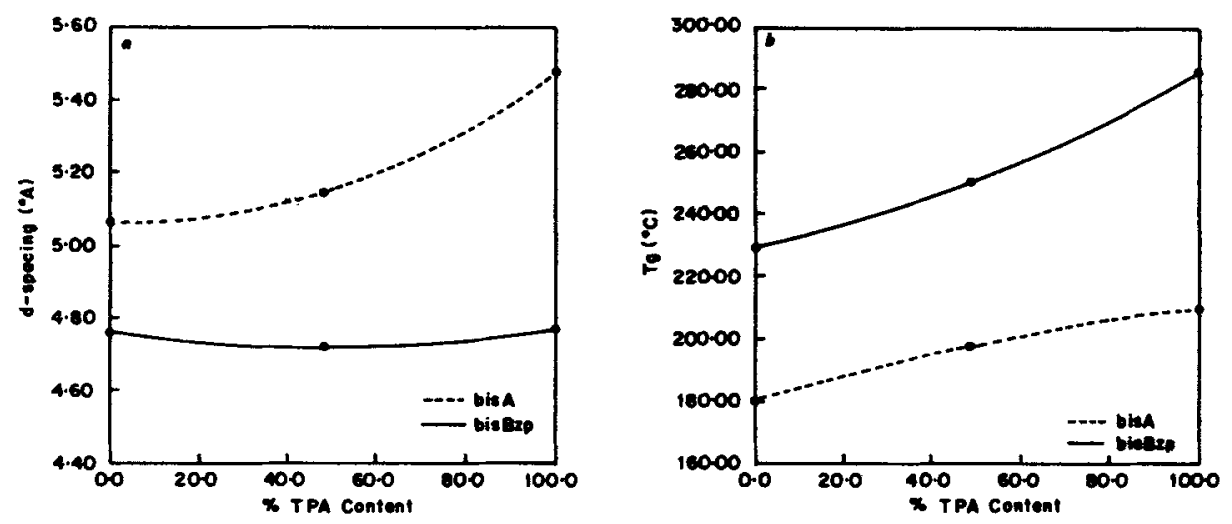

Figure 3. Variation of (a) d-spacing and (b) $T_{k}$ as a function of terephthalic acid content for polyarylates synthesized from iso- and terephthalic acid and bisA or bisBzp.

stronger in the case of bisBzp-I+T where, in spite of the reduced d-spacing, the increased chain stiffness still leads to higher permeability.

Table 1 shows the data for polyarylates prepared from bisBzp with isophthalic acid in addition to the $1: 1$ mixture of both iso and terephthalic acids. A common trend in polyarylates is that d-spacing, $T_{g}$ and permeabilities increase with increasing terephthalic acid content (Vetrivel et al 1991; Pessan and Koros 1993). Similar trends have been seen in isomeric analogues for polysulfones (Aitken et al 1990) and polyimides (Kim et al 1988; Coleman and Koros 1990). However the bisBzp based polyarylates are an exception to this empirical rule. The $T_{g}$ is increased; however, the $d$-spacing is constant and consequently the permeabilities are less affected by increasing terephthalic acid content in the bisBzp based polymers. It was not possible to measure permeabilities for bisBzp-T since this polymer is not soluble in common solvents. The difference in the d-spacing and $T_{g}$ variation with increasing terephthalic content in the bisBzp based polyarylates compared to bis $\mathrm{A}$ based polyarylates are shown in figure 3. Molecular modelling suggests that a change in chain colinearity, induced by the modified bisphenol, is responsible for erasing the well-known effect of the acid isomers on chain configuration and packing density.

\section{Conclusions}

While a previous study (Houde et al 1994b) showed that mono-substitution of the bisphenol bridge carbon did not significantly affect the polyarylate packing density, this study shows that disubstitution of this carbon is an effective way of changing the packing density (as measured by the d-spacing) of polyarylates. The d-spacing can be either decreased (by phenyl disubstitution) or significantly increased (by $-\mathrm{CF}_{3}$ disubstitution). As expected, the disubstitution also increases chain stiffness. Studies with bisBzp based polyarylates prepared with varying acid isomer ratios suggest that the bulky disubstituted groups alter the chain configuration. The variation in d-spacing, commonly seen with varying iso- and terephthalic acid ratios, is absent in these polyarylates. 
As expected from the increased $d$-spacing, bisF $-\mathrm{I}+\mathrm{T}$ has markedly increased permeabilities and reduced selectivities compared to the unmodified bisA based polymer. The increased permeability and selectivity of the bisBzp based polyarylates, in spite of the reduced d-spacing, is in support of the earlier finding (Houde et al 1994b) that decreased bisphenol phenyl ring mobility tends to increase the permeability.

\section{Acknowledgements}

We thank Drs B B Idage and J P Jog for the DSC data and Dr V Ramaswamy for the WAXD spectra. We thank the Council of Scientific and Industrial Research and the Department of Science and Technology (grant No. 323-1993) for financial support.

\section{References}

Aitken C L. Paul D R and Koros W J 1990 Proc. Int. conf. on membranes and membrane processes, Chicago, USA, vol $11 \mathrm{pp} 821-823$

Anand I N, Bales S E, Feay D C and Jeanes T O 1989 US. Patent 4, 840, 646

Barbari T A. Koros W J and Paul D 1989 J. Membrane Sci. 4269

Charati S G, Houde A Y, Kulkarni S S and Kulkarni M G 1991a J. Polym. Sci., Polym. Phys. Ed. 29 921

Charati S G, Houde A Y. Kulkarni S S and Kulkarni M G 199lb Macromolecules 252215

Coleman M R and Koros W J 1990 J. Meimbrane Sci. 50285

Houde A Y, Kulkarni S S and Kulkarni M G 1994a J. Membrane Sci. 95147

Houde A Y, Kulkarni S S, Kharul U K, Charati S G and Kulkarni M G 1994b J. Membrane Sci. (accepted)

Kim T H, Koros W J, Husk G R and O'Brien K C 1988 J. Membrane Sci. 3745

Light R R and Seymour R W 1982 Polym. Engg. Sci. 22857

Maruyama Y, Kakimoto $M$ and Imai Y 1986 J. Polym. Sci., Part A, Polym. Chem. 243555

McHattie J S, Koros W J and Paul D 1991 Polymer 32840

Morgan P W 1970 Macromolecules 3536

Muruganandam N, Koros W J and Paul D R 1987 J. Polym. Sci., Polym. Phys. Ed. 251999

Pace R J and Daytner A J 1979 J. Polym. Sci., Polym. Phys. Ed. 171675

Pessan L A and Koros W J 1993 J. Polym. Sci., Polym. Phys. Ed. 311245

Schmidhauser J C and Longley K L $1990 \mathrm{~J}$. Appl. Polym. Sci. 392083

Sheu F R and Chem R T $1989 J$ Polym. Sci., Polym. Phys. Ed. 271121

Stem S A, Mj Y, Yamamoto H and St. Clair A K 1989 J. Polym. Sci., Polym. Phys. Ed. 271887

Vetrivel R. Charati S, Kulkarni S S and Schultz J 1991 Polym. sci. contemp. themes, (ed.) S Sivram (New Delhi: Tata-McGraw Hill) vol Il p. 630 